\title{
Simple prompts reduce inadvertent energy consumption from lighting in office buildings
}

Article

Accepted Version

Tetlow, R. M., Beaman, C. P., Elmualim, A. A. and Couling, K. (2014) Simple prompts reduce inadvertent energy consumption from lighting in office buildings. Building and Environment, 81. pp. 234-242. ISSN 0360-1323 doi: https://doi.org/10.1016/j.buildenv.2014.07.003 Available at https://centaur.reading.ac.uk/37144/

It is advisable to refer to the publisher's version if you intend to cite from the work. See Guidance on citing.

To link to this article DOI: http://dx.doi.org/10.1016/j.buildenv.2014.07.003

Publisher: Elsevier

All outputs in CentAUR are protected by Intellectual Property Rights law, including copyright law. Copyright and IPR is retained by the creators or other copyright holders. Terms and conditions for use of this material are defined in the End User Agreement.

www.reading.ac.uk/centaur 
Central Archive at the University of Reading

Reading's research outputs online 


\section{Accepted Manuscript}

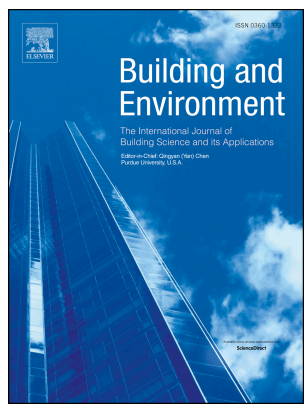

Simple prompts reduce inadvertent energy consumption from lighting in office buildings

Richard M. . Tetlow, C. Philip Beaman, Abbas A. . Elmualim, Kevin Couling

PII: S0360-1323(14)00219-4

DOI: $\quad$ 10.1016/j.buildenv.2014.07.003

Reference: $\quad$ BAE 3759

To appear in: Building and Environment

Received Date: 14 March 2014

Revised Date: 16 June 2014

Accepted Date: 2 July 2014

Please cite this article as: . Tetlow RM, Beaman CP, . Elmualim AA, Couling K, Simple prompts reduce inadvertent energy consumption from lighting in office buildings, Building and Environment (2014), doi: 10.1016/j.buildenv.2014.07.003.

This is a PDF file of an unedited manuscript that has been accepted for publication. As a service to our customers we are providing this early version of the manuscript. The manuscript will undergo copyediting, typesetting, and review of the resulting proof before it is published in its final form. Please note that during the production process errors may be discovered which could affect the content, and all legal disclaimers that apply to the journal pertain. 


\title{
Simple prompts reduce inadvertent energy consumption from lighting in office buildings
}

\author{
Richard M. Tetlow ${ }^{a^{*}}$ r.m.tetlow@ pgr.reading.ac.uk \\ C. Philip Beaman ${ }^{\mathrm{b}}$ c.p.beaman@ reading.ac.uk \\ Abbas A. Elmualim $^{c}$ a.a.elmualim@reading.ac.uk \\ Kevin Couling ${ }^{\mathrm{d}}$ kevin.couling@aecom.com
}

\footnotetext{
${ }^{a}$ Technologies for Sustainable Built Environments, University of Reading, UK

${ }^{b}$ School of Psychology \& Clinical Language Sciences, University of Reading, UK

${ }^{c}$ School of Construction Management and Engineering, University of Reading, UK

${ }^{d}$ AECOM, Bristol, UK
}

${ }^{*}$ Corresponding author:

Richard M. Tetlow

TSBE Centre, JJ Thompson Building, University of Reading, Whiteknights

PO Box 220, Reading, Berkshire, RG6

$6 \mathrm{AF}$

United Kingdom

Phone: $+44(0) 1183788533$

Fax: +44 (0) 1189313327

March 2014 


\title{
Simple prompts reduce inadvertent energy consumption from lighting in office buildings
}

\begin{abstract}
Building designs regularly fail to achieve the anticipated levels of in-use energy consumption. The interaction of occupants with building controls is often cited as a key factor behind this discrepancy. This paper examines whether one factor in inadvertent energy consumption might be the appearance of post-completion errors (when an intended action is not taken because a primary goal has already been accomplished) in occupants' interactions with building controls. Postcompletion errors have been widely studied in human-computer interaction but the concept has not previously been applied to the interaction of occupants with building controls. Two experiments were carried out to examine the effect of incorporating two different types of simple prompt to reduce post-completion error in the use of light switches in office meeting rooms. Results showed that the prompts were effective and that occupants switched off lights when leaving the room more often when presented with a normative prompt than with a standard injunction. Additionally, an over reliance on PIR sensors to turn off lights after meetings was observed, which reduced their intended energy savings. We conclude that achieving low carbon buildings in practice is not solely a technological issue and that application of user-models from human-computer interaction will encourage appropriate occupant interaction with building controls and help reduce inadvertent energy consumption.
\end{abstract}

Keywords: post-completion errors; energy performance; occupant behaviour; building controls; non-domestic buildings 


\section{Introduction}

Concerns surrounding anthropogenic climate change and energy security have compelled governments across the globe to impose strict targets for $\mathrm{CO}_{2}$ emissions abatement. The UK government has committed to an ambitious $80 \%$ reduction, on 1990 levels, by 2050 [1]. As the operation of non-domestic buildings is thought to be responsible for as much as $18 \%$ of total $\mathrm{UK} \mathrm{CO}_{2}$ emissions [2] it represents a key area where substantial reductions will need to be made if these targets are to be met. One of the main policies which the UK government is employing to stimulate reduction within the built environment is through the introduction of gradually stricter $\mathrm{CO}_{2}$ emissions targets in Part L building regulations. This has led to an increasing demand on the construction industry to deliver energy efficient buildings.

However, an established (and growing) body of evidence suggests that many supposedly energy efficient buildings do not, in practice, meet their intended levels of energy performance. In fact, associated $\mathrm{CO}_{2}$ emissions are frequently more than twice the design expectations [3], a discrepancy which has been termed the 'performance gap' [4]. This situation is unsurprising as typical energy modelling calculations focus on compliance with Part L building regulations which was never intended to consider the actual in-use performance of a building.

The actual energy performance of a building can be considerably influenced by the actions of the building users, for example the introduction of additional plug loads [5], the operation of building service controls [6,7], or failing to switch of lighting and equipment when not required [8]. In general the construction industry has considered the delivery of low carbon buildings to be largely a technological issue and has focused on reducing the performance gap through improving thermal performance, increasing the efficiency of building services, and incorporating low/ zero carbon technologies. 
From this viewpoint, occupants are often regarded as a hindrance to the building's performance and measures (such as automated controls) are taken to reduce the level of control that they have over their environment. For example, lighting accounts for around $16 \%$ of total electricity used in office buildings [9], so controls such as passive infra-red sensors (PIRs) are often employed to automatically switch off lighting when no occupancy is detected [10]. However, the results of occupant satisfaction surveys frequently indicate that their use in meeting rooms can be a source of occupant dissatisfaction as poorly calibrated PIRs will often inappropriately turn off lights during meetings [11]. A common alternative, to specify manual lighting controls for meeting rooms, provides users with an opportunity to adversely influence electricity consumption by leaving lights on when they are not required.

Efforts to reduce occupant related energy consumption in buildings either explicitly or implicitly subscribe to a 'rational choice' model [12] which assumes that people will both interpret information as intended and act rationally to modify their behaviour in line with this. Energy-reduction campaigns therefore encourage the deliberate conservation of energy rather than aiming to reduce inadvertent energy consumption. This somewhat simplistic approach to the behaviour of the building users, which ignores the automatic and habitual nature of much behaviour, has been shown to be incomplete at best [13] and ineffectual at worst [14, 15].

An alternative approach, adopted here, is to assume that building occupants are generally well-disposed towards energy conservation but are prone to inadvertent energy use from actions made (or not made) in error. Consider the common experience of sending an email and not realising that an intended attachment was not included until after the email is sent. This post-completion error [16] is a systematic, non-random error, a consequence of the routine, almost habitual, nature of the procedure, and the 
fact that the desired outcome (sending the email) is actually achieved before all the intended actions are accomplished. Further examples of errors of this type include leaving an original document on a photocopier, forgetting to replace the petrol-cap when refuelling a car, and failing to press "vote" after registering a preference on an electronic voting machine [17]. Such errors have been the subject of study in humancomputer interaction for a number of years [e.g. 16, 18]. However, the concept has not previously been applied to the interaction of occupants with building controls.

One reason for the neglect of post-completion errors in the study of the built environment is straightforward: in most studies of post-completion error there is an identifiable goal (the email is sent) embedded within a circumscribed task and the error can often be eliminated by restructuring the task and/or redesigning the relevant piece of machinery. For example, cash-machines (ATMs) dispense cash only after the user has retrieved their card. The goal (obtaining the cash) cannot therefore be achieved without first retrieving the card, eliminating this post-completion error [16]. Leaving the cash behind remains possible but is a rare occurrence because obtaining the cash was the goal of the transaction and - unlike leaving the card - forgetting the action which leads directly to the intended outcome only occurs under exceptional circumstances. Occupant behaviour within buildings, in contrast, is seldom so structured or as susceptible to analysis in terms of simple tasks with definable intended outcomes. The activities of occupants within non-domestic buildings can vary considerably, often in ways which could not be anticipated by the designers. Nonetheless, in the context of occupant use of building controls, post-completion errors could impact on building performance by increasing inadvertent energy use. For example, turning the lights on in a room in preparation for a meeting but then forgetting to turn them off as the room is vacated, or opening a window to ventilate a room but neglecting to close it afterwards. 
These basic features are common to almost all buildings, and reducing inadvertent postcompletion errors of these types could help to reduce the impact that the occupants have on energy performance.

In the studies reported here, we assume that failing to turn off an energy source - in this instance lights in a meeting room - is a post-completion error. Individuals are aware that to do so would be of financial and environmental benefit and it is a low-cost physical operation (literally, flicking a switch) however they fail to do so, in part, because once the meeting is over the "goal" is completed. Failing to switch the light off may therefore be a post-completion error rather than a simple consequence of poor motivation. As such, a simple visual reminder present at the point at which the room is vacated should significantly reduce the incidence of lights remaining on in empty meeting rooms, and such a reminder should remain effective over a period of time. Although such interventions are cheap and easy to implement - and are often done informally - it is perhaps surprising that their effectiveness over both the short and the longer-term has not previously been evaluated.

\section{Previous research findings}

\subsection{Occupant interaction with lighting controls}

There has been much interest in developing models to predict occupant interaction with lighting controls in single-occupant rooms, such as cellular offices. Hunt [19] and Love [20] proposed functions for the probability of occupants switching on lights based on illuminance level. They concluded that artificial lighting is more likely to be switched on when illuminance at the working plane drops below approximately 100 lux and once on it is unlikely to be switched off until the occupant leaves at the end of the working day, although the reasons for this were not clear. Pigg et al. [21] studied light switching 
behaviour in 63 cellular university offices with different lighting configurations including manual switching only and manual switching with PIR sensors to turn lights off when no occupancy was detected for a 10 minute period. Their results showed that length of absence (people were more likely to switch off lights when away for extended periods) and presence of PIR sensors (people were about half as likely to switch off lights when leaving a room with a PIR sensor than when leaving a room without one) were both strongly related to light-switching behaviour. These data suggest that, for cellular offices at least, not all failures to switch off lights are unintentional, as forgetting to do so is unlikely to be affected by these factors but there are also reasons to believe that this picture is incomplete.

A sophisticated and influential lighting algorithm called Lightswitch-2002 was developed by Reinhardt [22] to predict the probability of occupants switching lights on and off when arriving and leaving cellular offices. The algorithm was informed by field study data [23] and broadly collaborated Hunt's and Pigg et al.'s findings. In related work, Fabi et al. [24] also drew a distinction between 'active' and 'passive' situations for occupant interaction with lighting controls and they proposed different models for these different situations, Results from their field study showed that a range of environmental variables had a significant impact on light-switching behaviour including different illuminance levels and ratios within the room, but also indoor temperature. Interestingly, Fabi et al. also suggested that occupants are generally less likely to switch lights off than on because they are cued to switch lights on by poor visual comfort whereas there are no analogous cues for switching lights off. This result is consistent with the idea that providing appropriate visual cues to switch off lights will reduce the number of lights left on unnecessarily. 
Since the majority of these studies were carried out in cellular offices (i.e. with one or two occupants) and our studies will take place in shared meeting rooms there may also be significant differences between the way in which occupants perceive their relationship with their environment and this may also influence the number of “inadvertent" errors resulting in lighting being accidentally left on. Slater et al. [11] suggested that occupants will use lighting controls differently depending on the amount of 'ownership' they can be considered to have over a particular space. They proposed six separate levels for ownership ranging from 'owned' (i.e. cellular offices) to 'unowned' spaces (i.e. circulation areas). Slater et al. classify meeting rooms as 'temporarily owned' spaces and suggest that lights are often left on in these areas as no one feels directly responsible for them. Occupant interactions with light switches in meeting rooms (or temporarily owned spaces) have not been explicitly studied previously.

\subsection{Post-completion errors}

In a series of studies, Chung and Byrne $[25,26]$ demonstrated that post-completion errors can be completely eradicated through presenting users with visual prompts. These prompts had three key features: salience (the cues stood out visually), specificity (they indicated precisely and unambiguously what operation was required), and timeliness (they appeared right at the moment the action needed to be taken). Of these features, Byrne [27] later determined that timeliness (being just-in-time) was the most important and salience the least important. This implies that a reminder to take an intended action which appears at the appropriate moment may have a substantial impact upon a postcompletion error. It is also important to consider the longevity of behavioural interventions, an aspect that is rarely examined in the literature. Ament et al. [18] showed that, after repeated exposure to visual cues, errors were not reinstated when the 
cue was no longer available. This suggests that the impact of such a reminder on individuals exposed to it may persist over a relatively long period.

\subsection{Social norms}

A further, often underappreciated, feature of human-building interaction is that occupants interact with the building both as individuals and as (more or less) cohesive social groups. Social norms are the behavioural expectations present within a specific group. The tendency to conform to these implicit expectations is often unconscious and people routinely fail to realise the extent to which social norms have influenced their behaviour [28]. In a study of domestic energy consumption, Schultz et al. [29] informed around 300 households in California about how much energy they were using relative to the average of households across their neighbourhood. As a result households who were above the average consistently lowered their energy consumption. Interestingly, a 'boomerang' effect occurred where households that were using much less than the average actually increased their usage towards the average. This 'boomerang' effect was reduced by the inclusion of simple emoticons (image of a smiley or sad face) indicating whether the current energy usage was socially approved or disapproved. Social norms have also been shown to be successful in promoting pro-environmental behaviour in the hotel and catering sector [30], but their application has not been so fully explored in offices which clearly have very different physical and social conditions. The approach we have taken is to add social cues to prompts to switch off lights when leaving a meeting room, which also goes beyond [29] by explicitly linking the social cue to a reminder to perform a single action (switching off the light) and beyond [30] by examining the long-term effect of such cues. It is important to determine whether simply framing information in terms of the behaviour of other group members increases compliance with the environmental message of the prompt by (consciously or 
non-consciously) impacting upon the perceived social necessity of avoiding the postcompletion error.

\section{Experiments - reducing post-completion errors}

Two different types of prompt were created to encourage the users of two different office buildings to turn off lights when exiting meeting rooms. One was a standard pictorial prompt, the other contained a normative cue. Their design and placement was such that they satisfied Chung and Byrne's $[25,26]$ three key aspects for an effective visual cue; salience, timeliness, and specific to the required action. Employees in both buildings were from the same engineering consultancy and were not considered to be energy experts. They were given no prior information about the appearance or the meaning of the new prompts. The prompts were placed in four meeting rooms in each building over different monitoring periods and their effect on lights being switched off was recorded. The expectation was that both types of prompt should increase the likelihood of lights being switched off by reducing the post-completion error. We also anticipated that the normative prompt should be more effective than a straightforward injunction to turn off the lights due to its additional social norm information.

Initially baselines for each room were established by determining the frequency that employees turned lights off after meetings prior to any intervention. This provided basic data on the extent to which energy was wasted after meetings. Experiments 1 and 2 use different buildings, with the lighting for the meeting rooms in Experiment 1 wholly controlled by manual switches. In contrast, the lighting for meeting rooms in Experiment 2 is additionally under the control of PIR sensors. Although this was not a primary motivation of our study, this difference enables us to compare the extent to which manual switches are used when occupants are aware that lighting is also under automatic control. 
In Experiment 1, we additionally examine the effect of the number of people leaving the room at any one time. In an idealised situation the larger the group the more likely someone is to switch off the lights upon leaving the room. However, there is a possibility that the presence of others within the group might actively inhibit individuals from carrying out this action, possibly to the extent that larger groups are more likely to leave the lights on. Although an oversimplification, the assumption that each member of the group will be uninfluenced by the presence of others could be described by the model $(1-o)^{n}$, where $o$ (for off) is the simple probability that any individual within the group will switch off the lights, independent of the value of $n$ (i.e. the number of others within the group). So, for example, if the probability $o$ that a single individual habitually extinguishes a light is .9 then the probability that they fail to do so $(1-o)$ is .1 . The probability that two individuals independently fail to switch off the light is then $.1^{2}$ or .001 . Hence, the probability that the light remains on must therefore be .999 . It is apparent from this that if the chances of any individual extinguishing the light as they leave the room are of any magnitude, then unless something about the situation inhibits individuals from switching off lights as they leave, the probability that a light is left on when a large group leaves the rooms must soon become vanishingly small.

The extent to which the observed behaviour deviates from this idealised situation indicates how people deviate from the commonly held assumption about human behavior that individuals will react consistently and independently of the activities of others. In reality, if occupants positively influence each other, then the rate of increase should be greater than that predicted by the model. If, however, the presence of others reduces the probability that any one individual switches off the light, then the rate of increase should be slower than that predicted by the model, the proportion of times that the light is switched off may remain constant, or even decrease. Previous 
effects of providing social norms were observed in an environment (e.g., within hotel rooms) where there were few alternative social cues available to influence behaviour (the actions of guests in other rooms were not observable). On leaving a room, the social cues of other members of the group not obviously switching off a light as they pass by might inhibit individuals from acting themselves, a phenomenon referred to in the social psychology literature as distributed responsibility.

\subsection{Materials \& design}

Prompts were sized at $120 \mathrm{~mm} \times 120 \mathrm{~mm}$. The first prompt was a standard pictorial prompt. The second prompt had the same format with the only alteration being that the picture of a light bulb was substituted for a representation of a smiling figure with a percentage indicating the amount of people who may have previously turned off the lights when leaving the meeting room (see Figure 1). This percentage was an arbitrary value based around the $75 \%$ value utilised in Goldstein et al. [30] and was slightly altered between each of the meeting rooms in the two different offices. An arbitrary figure, rather than dynamically-updated feedback, was chosen as the simplest and most cost-effective means of testing the utility of social norms for providing positive encouragement but in principle a regularly updated figure could be used the same way.
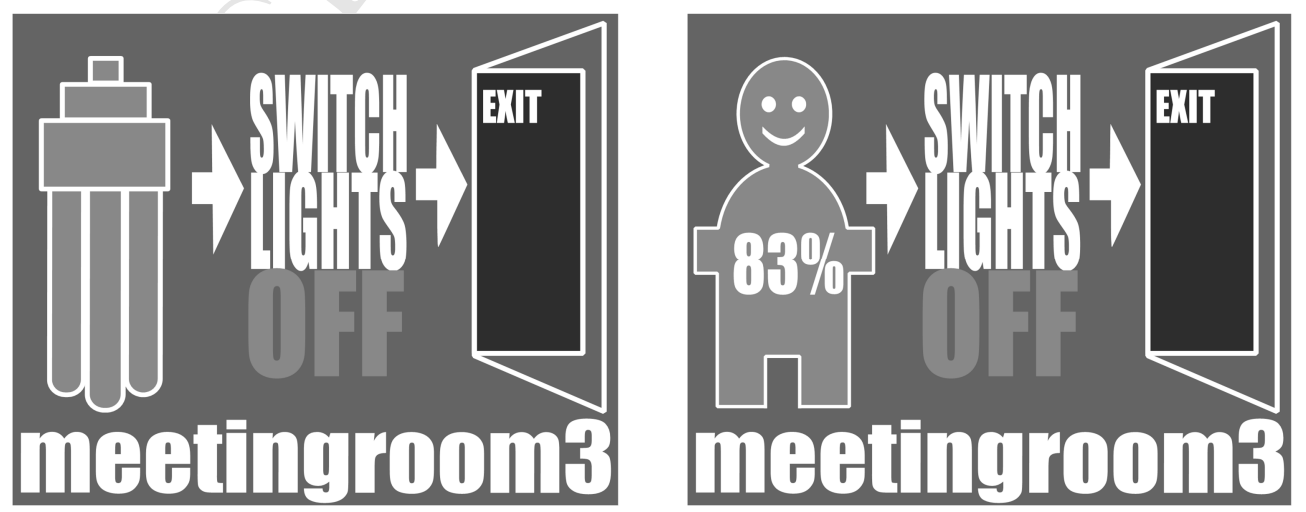

Figure 1. Example of standard prompt (left) and normative prompt (right). 


\subsection{Experiment 1 - Longevity and distributed responsibility}

\subsubsection{Participants}

Employees leaving four different meeting rooms in Portwall Place in Bristol between the standard working hours of 9am - 5.30pm were observed on 149 occasions during the initial ten working day observation period and on 122 occasions for the ten working day intervention period.

\subsubsection{Procedure}

Meeting rooms were designed with two glass walls facing onto open plan office areas to allow ingress of light but had no external windows. The doorways were located in one of the full height glass walls. Consequently the light switches were located on the solid walls behind the doors (see Figure 2). Each meeting room already contained an existing text-based prompt located above light switches stating 'Please turn off the lights when you leave, thanks'. Analysis of this existing prompt in terms of Byrne's suggested requirements for a key visual prompt suggested that it would be largely ineffectual because it was insufficiently salient (it comprised only of small (12pt) monochromatic text) and its timeliness was poor (it was located above the light switch behind the door so was not immediately obvious upon vacating the room). Initial monitoring to confirm this impression was carried out for a ten work day period from the researcher's desk which provided a good view of all meeting rooms. Employees were unaware that they were being monitored. After a baseline was established the new prompts were introduced. These were positioned on the glass walls adjacent to the opening side of the door, between eye level and the door handle, the existing text based prompt was left in place. Two normative prompts and two pictorial prompts were placed in the four different meeting rooms. Incidences of turning off the lights were again recorded. After 
five days the prompts were swapped between rooms. Prompts were then left in meeting rooms for a six month period prior to observations recommencing. Again monitoring was carried out for a ten work day period.
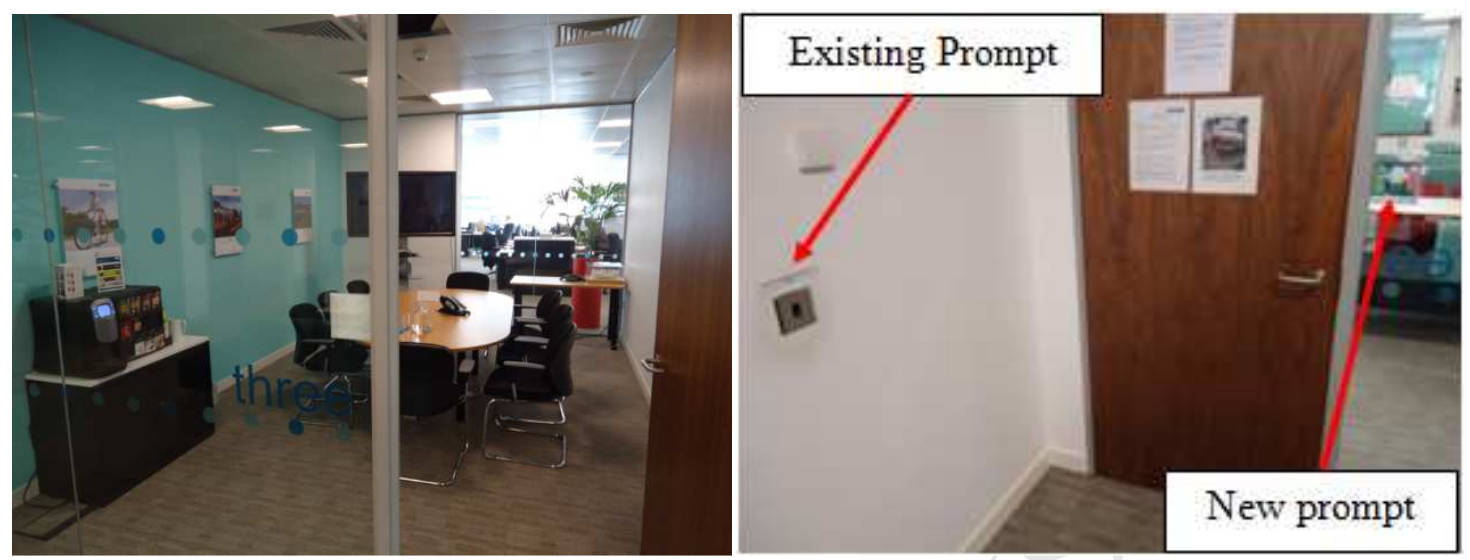

Figure 2. Typical meeting room at Portwall Place (left) and location and relative size of new prompts and pre-existing reminders (which remained in place in all conditions) (right).

\subsubsection{Results}

Figure 3 displays the observations for all meeting rooms over all conditions and shows that when larger groups met within the rooms, the proportion of lights being switched off was lower, the opposite pattern from that predicted by the independent responsibility assumption, but consistent with the idea that distributed responsibility increases with group size, reducing the likelihood of an individual turning off the lights. Expected percentage in this figure refers to the percentage expected on the basis of the calculation in the idealised model proposed in Section 3. Data inputted for the idealised model are included in Table 1. 


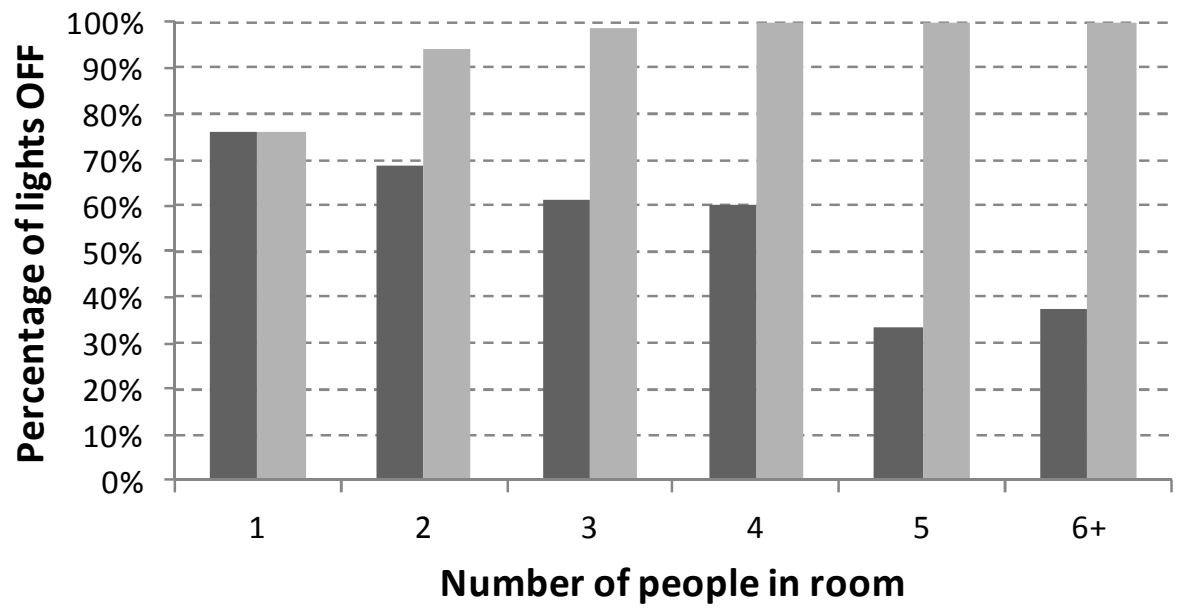

Observed percentage Expected percentage

Figure 3. Bar chart showing the expected percentage of lights turned off and the obseved percentage of lights turned off for the number of people across all rooms and conditions.

Table 1. Data used for calculation of observed and expected percentages of switching lights off in idealised situation model. The observed probabilty of a single individual turning off a light when only only one person was present in the room was used as the basis for the $o$ value of the model and figures are rounded to two decimal places (i.e., $1-\left(.24^{2}\right)=.94$ and so on).

\begin{tabular}{ccccc}
\hline $\begin{array}{c}\text { Number of } \\
\text { people in room }\end{array}$ & $\begin{array}{c}\text { Total number } \\
\text { of meetings }\end{array}$ & Lights off & $\begin{array}{c}\text { Observed } \\
\text { percentage }\end{array}$ & $\begin{array}{c}\text { Expected } \\
\text { percentage }\end{array}$ \\
\hline 1 & 25 & 19 & $76 \%$ & $76 \%$ \\
2 & 51 & 35 & $69 \%$ & $94 \%$ \\
3 & 31 & 19 & $61 \%$ & $99 \%$ \\
4 & 5 & 3 & $60 \%$ & $100 \%$ \\
5 & 6 & 2 & $33 \%$ & $100 \%$ \\
$6+$ & 8 & 3 & $38 \%$ & $100 \%$ \\
\hline
\end{tabular}

Data were analysed using a chi-squared $\left(\chi^{2}\right)$ test which is used to determine whether distributions of categorical data (e.g., the numbers of people turning the light off in meeting room 1 vs. the number of people turning the light off in meeting room 2) significantly differ from one another (see [31] for a good introduction to statistical tests for behavioural studies). The conventional $5 \%$ level for statistical significance was applied and the test was a 1-tailed (or directional) test because we are interested in whether prompts are more effective than no prompts, not vice versa. A $5 \%(p=.05)$ level 
of significance indicates that the data observed would only occur $5 \%$ of the time if the null hypothesis were correct. Our null hypothesis was that the prompts would have no effect on the likelihood that lights would be turned off after a meeting had finished and this is rejected with $p<.05$. We conclude that the observed advantage for displaying prompts is unlikely to have appeared because of chance factors alone. Results for the initial period of observation over the three different prompt conditions are shown in Figure 4, which indicates that in $86 \%$ of cases the lights were turned off in the most effective, normative prompts, condition compared to $56 \%$ of the time in the least effective, control condition. There was a statistically significant effect of introducing the prompts, $\chi^{2}(2)=6.79, p<.03$. However, further analysis showed no statistically reliable effect of normative prompts over standard prompts $(p=.24$, using Fisher's exact test (which gives a more accurate calculation for $p$ than chi-squared if small numbers of observations are involved)).

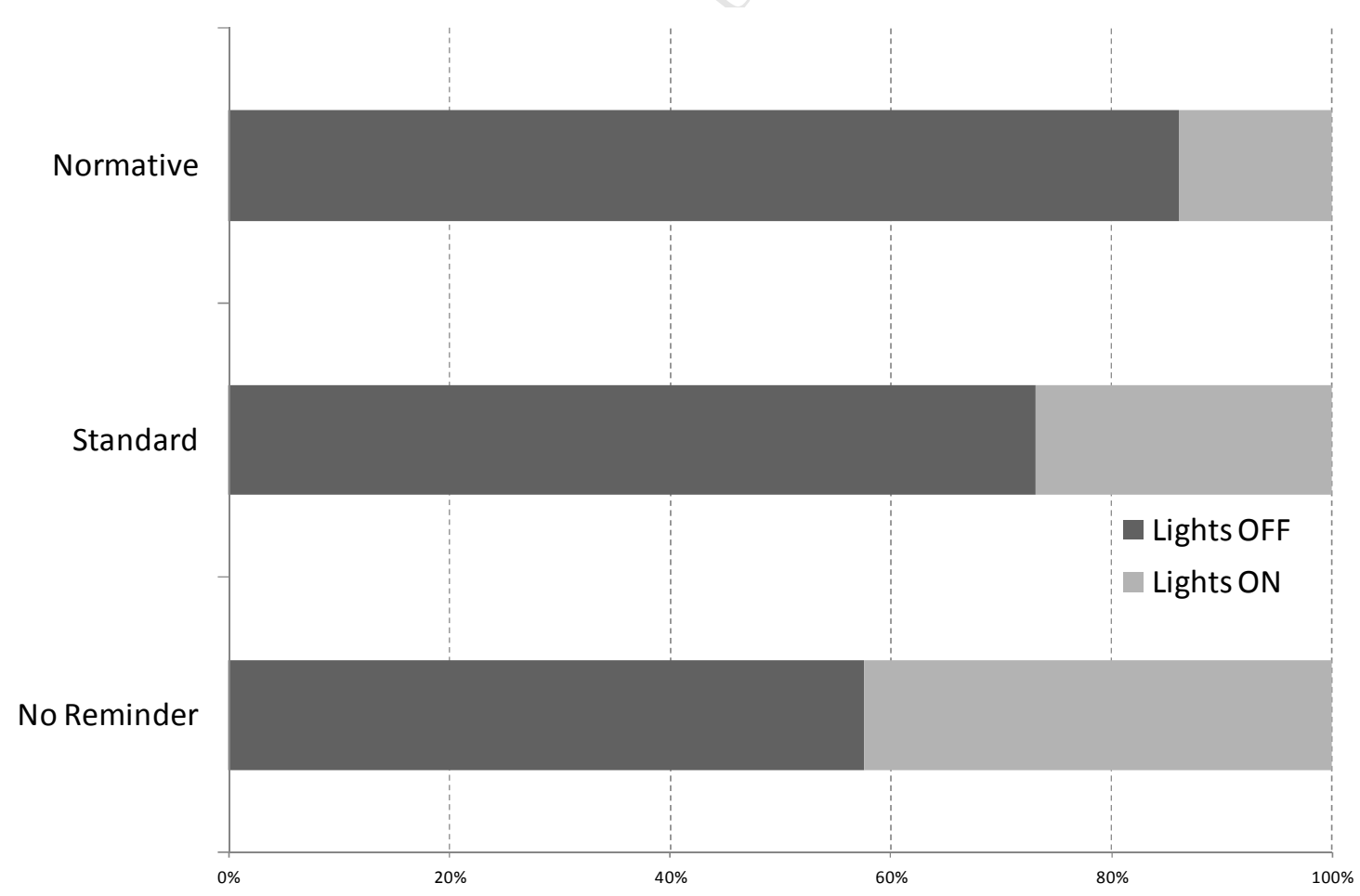

Figure 4. Stacked bar chart showing percentage of people turning lights off or leaving them on when exiting meeting rooms for each prompt condition during initial period of observation. 
Results for the period of observation after six months are displayed in Figure 5.

Here the two prompt conditions were combined as no differences were found between them during earlier analysis and as at this point we were only considering differences that may have emerged over time, not in differences between prompt types. Analysis of these results show that a statistically significant effect of the prompts being present remains, $\chi^{2}(1)=15.64, p<.05$. The observations after six months were also compared to the initial observations of both prompt conditions combined (pictorial plus normative) but this result was not significant, $\chi^{2}(1)=0.42, p>.05$. There is therefore no statistical evidence that the effect of the prompts declined over this six month period.

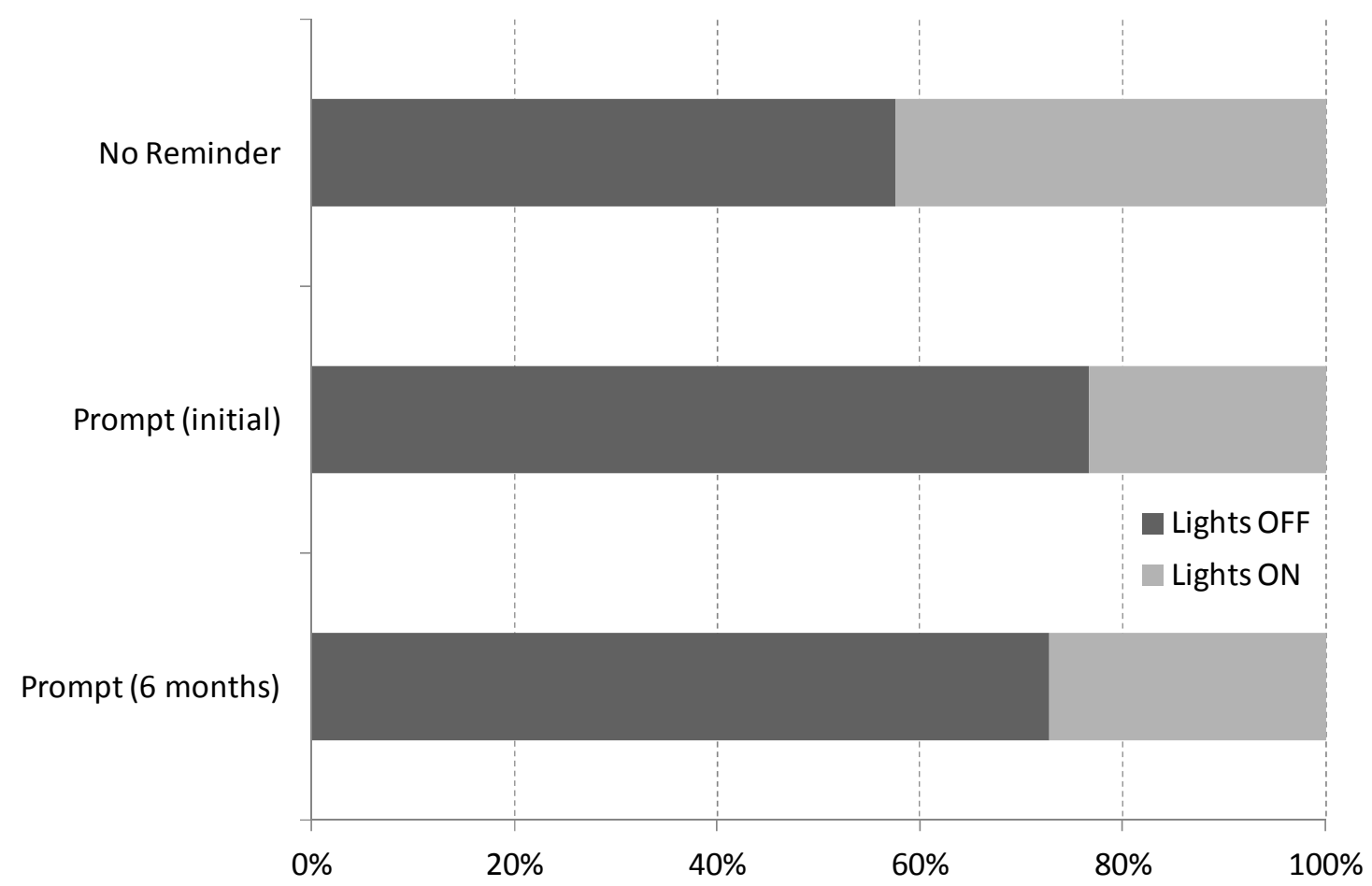

Figure 5. Stacked bar chart showing percentage of people turning lights off or leaving them on when exiting meeting rooms for prompts initially and prompts after 6 months.

\subsubsection{Discussion}

The results of Experiment 1 are informative in several ways. Firstly, they show that - in rooms with manually controlled lights - the number of people attending any meeting had a negative impact on the chances of the lights being turned off when the occupants leave. It should be noted though that because the majority of observed meetings had 
fewer than 4 people the sample size for the meetings with more than 4 people is relatively low. However, it raises the possibility that smaller groups are more likely to switch off lights than larger groups and is a factor that may need consideration in the future development of light-switching behavioural models. It is difficult to formulate a coherent policy for building designers and managers on the basis of this observation alone (clearly, it is not possible to legislate for the number of people present at meetings) but it is nevertheless worth noting that larger rooms where meetings are attended by more people are likely to remain lit unnecessarily after the meeting has ended. In determining whether - and where - to install PIRs, building managers may wish to take this into account. Secondly, the baseline data indicate that lights remained on after meetings were concluded in slightly less than half of the observed occasions, resulting in wasted energy. Thirdly, the impact of a simple visual reminder was statistically significant. After six months, the presence of a reminder was still reducing the incidence of lights being left on to a statistically significant degree. Given the low cost of such an intervention, it can be recommended for most building managers provided the key criteria for such reminders of salience, specificity and timeliness are met.

The social pressure implicit within a "normative" reminder failed to have any impact over and above the basic effect of a reminder however, which is inconsistent with previous reports of pro-environmental behaviour being encouraged by appeals to social norms [29, 30]. It is tempting to suggest that, based on the results of Experiment 1, that social norms do not aid energy conservation in the office environment where individuals are operating as paid employees rather than as householders (possibly with responsibility for the utility bills) or as (probably paying) hotel guests. However, it is also possible that the effect size of prompts framed with normative information is 
smaller than originally considered, in which case it would be premature to dismiss the use of social norm prompts in this way. A number of factors militate against a social norm prompt being as effective as previously demonstrated by other studies without necessarily being completely ineffective. One obvious factor is that, in contrast to these situations where the energy consumption of friends and neighbours - or their behaviour in adjoining hotel rooms - is unlikely to be known, the people observed in this study may well have been aware that their own experience of other people switching off lights when leaving meeting rooms differed from the figures presented on the "normative" prompt. Indeed, the mere fact that in larger groups any one individual seems to become less likely to extinguish a light when leaving implies that on many occasions these individuals will have seen groups of others leaving the rooms without switching the lights off. Under such circumstances the "normative" prompt may lose much of its force. Nonetheless, given that there is no extra cost to providing a reminder based around social norms rather than a standard injunction, the numerical (but statistically unreliable) advantage observed for the "normative" prompt is worthy of further investigation. The method employed in Experiment 1, of an observer physically present to record the behaviour of groups leaving meeting rooms, limited the number of observations that could be made and so the study was not particularly statistical powerful to detect smaller to medium-sized effects which could translate into substantial energy savings over time. To this end, Experiment 2 repeated the investigation, using automatic data loggers to record occupancy and incidences of lights being turned off, in order to increase the sample size and hence the statistical power.

\subsection{Experiment 2: Normative prompts}

Experiment 1 was conducted across rooms in which no data-loggers were installed because it allowed us to examine the effect of group size (Figure 3) uncontaminated by 
any expectations amongst the group that lights would be switched off automatically.

The main research question for Experiment 1 was answered by the positive, statistically significant, impact of the prompts but the lack of statistical power in the study meant that it was difficult to form any valid conclusions about the effect of the social norm information. Guided by the results of the previous studies, we assumed that this would be a larger effect than that observed in Experiment 1, the results of which are consistent with either no effect or a much smaller effect than we presumed. Experiment 2 resolves this ambiguity by replicating the manipulation of Experiment 1 but with a more statistically powerful design. This required automated data collection in meeting rooms of similar size and design and with employees from the same engineering consultancy. For this to be possible Experiment 2 was conducted in rooms with PIRs already installed. This has two incidental benefits. It will test whether the pattern of data observed in rooms without PIRs is replicated in rooms with PIRs (and hence whether the results generalise across different offices with potentially different occupant expectations) and whether the existence of PIRs (which occupants are not explicitly informed about but the action of which they will readily observe over time) impacts upon overall light-switching behaviour. Note that any differences between Experiments 1 and 2 in this regard will be the outcome of cross-experiment comparisons and therefore such data should be treated with caution, but nonetheless any prominent trends or contrasts will be of interest.

\subsubsection{Participants}

The experiment was repeated at a separate office building (Mid City Place in London) using automatic light and occupancy data loggers. This was considered appropriate as the statistical power of Experiment 1 was limited due to the small sample size obtained through direct observation. To obtain an experimental power of 0.8 , Cohen [32] 
recommends a sample size for a $\chi^{2}$ analysis of at least 964 (assuming a small effect size). Meeting rooms in Mid City Place were monitored between working hours of 8am to $8 \mathrm{pm}$. Periods of occupancy and whether the lights were on or off were recorded for the three conditions. In total, for the baseline condition 319 meetings were recorded, for the standard prompt condition 388 meetings were recorded, and for the normative prompt condition 422 meetings were recorded. Readings for Saturday and Sunday were not recorded.

\subsubsection{Procedure}

Similarly to meeting rooms in Portwall Place, meeting rooms in Mid City Place had doorways located in full height glass walls with the light switches behind doors (see Figure 6). However, three of the meeting rooms had external windows. The manual light switches were also slightly more complicated allowing the user to select multiple lighting levels by operating ten individual buttons. There was no existing prompt in place to encourage occupants to turn the lights off when exiting. Lighting in these meeting rooms was additionally controlled by a PIR sensor located in the ceiling; if no occupancy was detected for a period of 20 minutes lights would be automatically turned off.
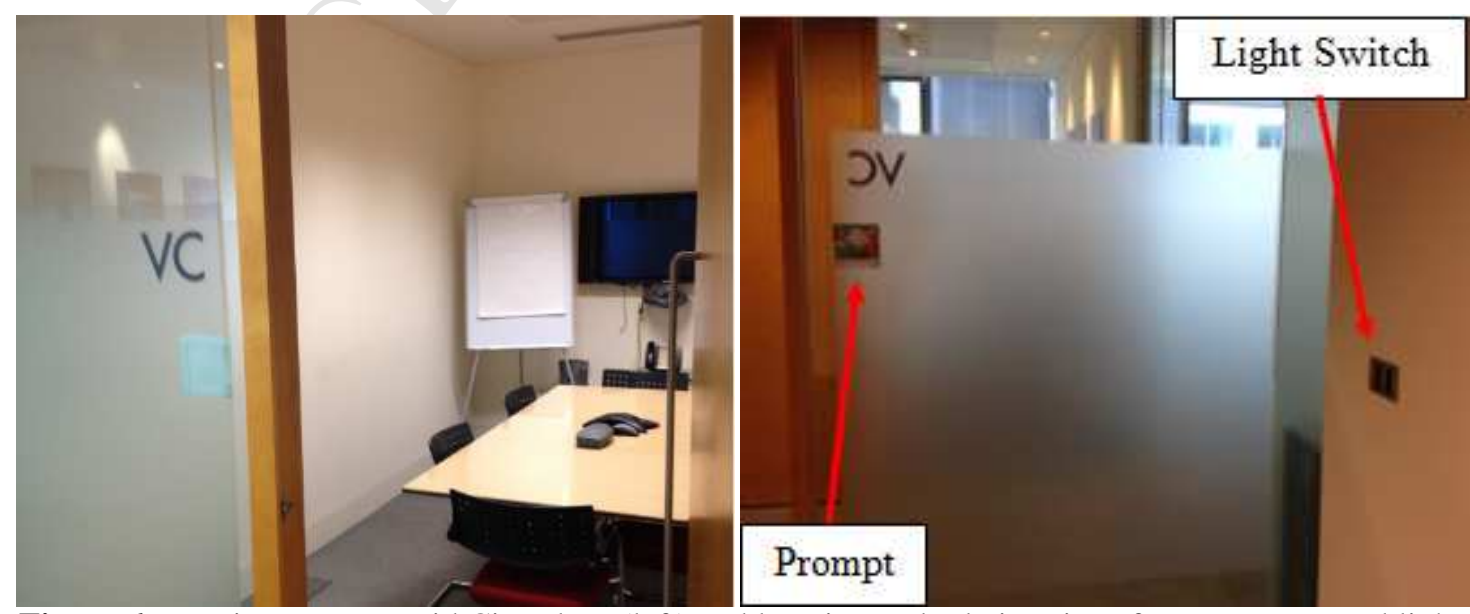

Figure 6. Meeting room at Mid City Place (left) and location and relative size of new prompts and light switch (right). 
$\mathrm{HOBO}^{\mathrm{TM}}$ Occupancy/ Light data loggers (UX90-005x) were attached to the ceiling of meeting rooms using magnets. Where the room had an external window, fibre optic pipes were used to direct the data logger's light sensor towards one of the room's main light fittings, to minimise the chance of natural light inadvertently influencing readings. The data loggers monitor whether lights were on or off and whether the room was occupied or not and record any change of state in either of these variables. Initial monitoring for the baseline condition in the four meeting rooms was carried out for an eight week period. After the baseline was established the prompts were introduced. Again, these were positioned on the glass walls adjacent to the opening side of the door, between eye level and the door handle. Two normative prompts and two pictorial prompts were placed in the four different meeting rooms for an eight week period. After this period, the type of prompt (i.e. normative or standard) in each room was swapped and monitoring continued for further eight weeks.

Although the use of automatic data loggers allows for a larger sample size a certain amount of interpretation must be applied to the data. Direct observations prior to the study indicated that few meetings held in the monitored rooms lasted for less than 30 minutes with employees preferring to use the less formal break-out areas for such encounters, and there were occasionally very short turnaround periods between one meeting finishing and a new meeting beginning. To interpret the data therefore, two rules were applied to reduce any "noise" (e.g. cleaners coming in at the end of meetings or the data logger's PIR sensor repeatedly detecting occupancy for only short periods - a situation which can occur when the room is lightly occupied): A 'meeting' was only recognised as such if occupancy was logged, uninterrupted, for at least a 30 minute period and the room was unoccupied for at least 2 minutes before a meeting was judged to have started. 


\subsubsection{Results}

The null hypothesis was that the prompts would have no effect on the likelihood of lights being turned off after meetings. Results for the four meeting rooms over the three different prompt conditions are shown in Figure 7. There was a statistically significant effect of introducing the prompts, $\chi^{2}(2)=12.41, p<.05$. Further analysis showed that there was also a statistically significant difference between the two types of prompt (normative and standard), $\chi^{2}(1)=4.61, p<.05$ and Fisher's Exact test, $p=.0213$ (1tailed). Therefore there is significant evidence to reject the null hypothesis and accept the alternative hypothesis that the normative prompt increases the chance that people will turn the lights off in meeting rooms.

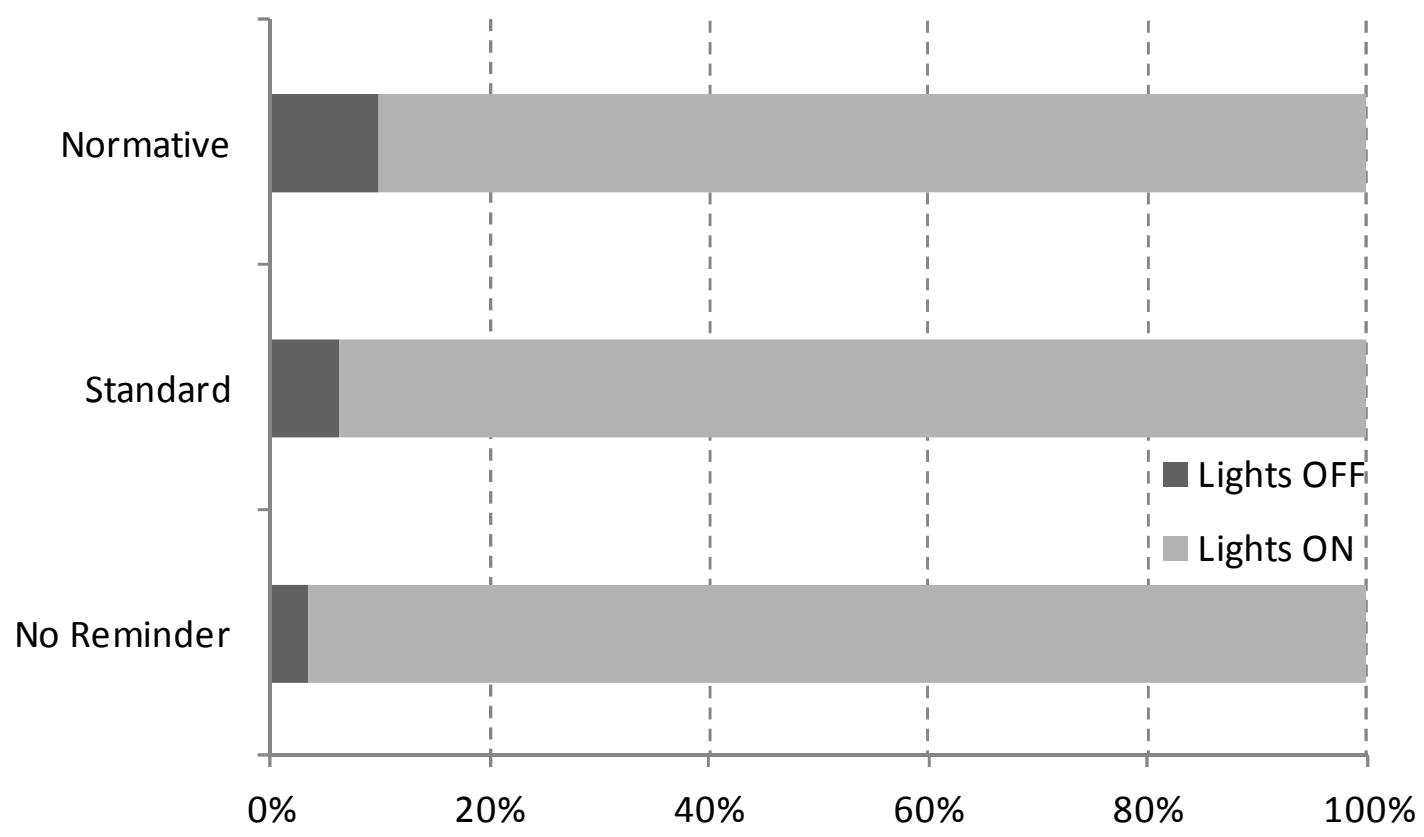

Figure 7. Stacked bar chart showing percentage of people turning lights off or leaving them on when exiting meeting rooms for each prompt condition.

\subsubsection{Discussion}

Reinforcing findings from Experiment 1, results from Experiment 2 indicate a greater incidence of lights being switched off after meetings had completed once the prompts had been introduced. Additionally, and consistent with our original hypothesis, they 
show a significant difference between the two types of prompt with the normative prompt being more successful at motivating the occupants to switch off lights after meetings had been completed. However, the effect of the additional social norm information was smaller than previous studies have indicated. As there are no additional time or cost implications associated with the use of normative prompts as appose to standard prompts it can be recommended as a simple measure for building managers to motivate energy conservation.

\section{General discussion}

The results from the experiments described here demonstrate that increasing the saliency, timeliness, and specificity of a visual prompt (or indeed introducing a new prompt) significantly increased the likelihood that occupants would turn off lights after meetings had concluded. These findings suggest that a post-completion error can indeed occur during an occupant's interaction with manual light switches in that the terminal step of switching off lights can often be forgotten as the primary goal of holding the meeting has already been completed.

Although many modern office lighting installations will be automatically operated with PIR sensors, there is evidence to suggest that automatic lighting in meeting rooms is an area of potential occupant dissatisfaction. In this study the meeting rooms in Mid City Place (Experiment 2) had PIR sensors to automatically turn lights off when no occupancy was detected after a period of 20 minutes. Comparison of meeting rooms to those in Portwall Place (where there were no PIRs, Experiment 1) indicate that the inclusion of PIRs may mean that the occupants are much less likely to use light switches; the baseline analysis revealed that only 3\% of meeting in Mid City Place ended with lights being manually switched off compared to 58\% in Portwall Place, a difference much larger than that between the prompt and no-prompt conditions in either 
office. This is an important point and it should be noted that the employees in Experiment 2 are not explicitly told that there are PIR sensors installed to automatically turn off lights. Regardless, the design intent of PIRs to control lighting is often to act as an 'insurance' so if the occupants forget to turn off lights then they are automatically switched off, not that occupants should rely on them to turn lights on and off all the time. The marked contrast between the overall levels of lights being switched off across the two Experiments is therefore informative. These results are consistent with Pigg et al. [21] who evaluated the effectiveness of PIR sensors in switching lights off in cellular offices at a US university. They discovered that the inclusion of PIR sensors to automatically turn off lighting led to perceptible changes in the behaviour of the occupants. Specifically they were less likely to manually switch off lights when leaving their office, preferring instead to rely on the PIR to do this for them. Pigg et al. estimated that this behavioural shift led to a reduction in potential energy savings from the PIR in the region of $30 \%$. The findings from our experiments support this. Analysis of the data from Experiment 2 reveals that the likelihood of either the PIR sensor or an occupant switching the lights off immediately after meetings (across all four rooms) was just $13 \%$. This clearly compares unfavorably with the $58 \%$ chance that an occupant would turn the lights off after a meeting in Portwall Place where there is no PIR sensor. Energy costs are therefore being incurred wholly unnecessarily during the interval between occupants leaving the room and the PIR switching the lights off on every occasion that this happens, costs which may accumulate over time.

Interestingly, results from the data loggers also indicate that the PIR sensors would routinely fail to turn the lights off after the 20 minute period and they could be on for a number of hours with no actually occupancy detected. This was indeed found to be the case during a site visit on a weekend when lights in some meeting rooms were on 
even though no employees were present. It is likely that the positioning of the PIR sensor meant that any movement in the adjoining corridor (particularly if the door was left open) could be misinterpreted as an occupied room resulting in the lights remaining on for an additional 20 minutes. This situation was probably exacerbated by the design of the meeting rooms with a glass wall facing on to the corridor through which the infrared light was found to pass ${ }^{*}$. This highlights the need for suitable placement of PIR sensors as well as ensuring they are not over sensitive during the calibration process.

Although this paper has focused on the interaction between occupants and light switches it is likely that similar errors are occurring with the use of other building control systems. For instance, window opening for natural ventilation and night cooling, the use of programmable thermostats, and failing to turn off small power devices when they are not in use. In the past the misuse of control systems has led designers to regard the occupants as a nuisance whose ability to interact with building services should be severely limited. However, the evidence now suggests that restricting the amount of control occupants have over their environment can negatively impact on health and satisfaction [4] and to some degree energy consumption [33]. This, coupled with a returning interest in natural and mixed mode ventilation and night cooling strategies, is presenting occupants with an increasing level of control over their environment. Designers now face a clear problem; providing opportunities to interact with the building and its systems whilst considering the likelihood that the occupants will use them inappropriately.

The research presented here has clear implications for design teams. As the detail of control systems feature relatively late in the design and construction process

\footnotetext{
* Typically, glass is designed to pass visible wavelengths but to block infrared in order to retain heat. However, shorter wave infrared can often pass through, which was the case here.
} 
they are particularly vulnerable to value engineering (where a specified system is substituted for an inferior version on the basis of a cost saving). These findings emphasise the need to ensure that clear and intuitive control systems are not only specified but are actually installed and that the salience, timeliness, and specificity of those control systems are adequately considered during the design stage.

\section{Conclusions}

When building occupants use manual light switches a post completion error can occur resulting in inadvertent electricity consumption. This post completion error can be greatly reduced by introducing a visual prompt to turn lights off as an occupant exits a room, which is salient, timely, and specific. Additionally, these prompts can be made more effective by framing them in terms of social norms rather than the standard injunctions to turn off the lights which are a staple part of any employee engagement campaign. It is likely that similar, routine and predictable, errors are occurring during many interactions between occupants and building controls and that this is contributing to the performance gaps that are frequently discovered during building performance evaluations. The inclusion of automated controls does not always achieve the expected savings in real world situations as the occupants may (unconsciously) modify their behaviour in response to them. For example, if PIRs are implemented to reduce energy consumption for lighting but occupants use them as an alternative to switching off lights then the period between the occupant leaving a room and the PIR responding to this change results in energy wastage. Similarly, occupants opening windows instead of adjusting air-conditioning in response to perceived excess heat is not usually an energyefficient reaction. This highlights that it is futile to integrate ever more complex technologies within building designs if the occupants are liable to make routine errors when interacting with them. Designers often claim that the problem is not with the 
design of the systems, but with people operating them incorrectly. However, it is important to emphasise that systems are created with the implicit purpose of allowing the users to perform necessary functions; they are not designed to operate as entities in themselves. If the majority of users cannot operate the system easily and consistently to achieve their desired purpose then the design has failed. Delivering energy efficient buildings is not exclusively a technological issue and the construction industry needs to develop a more sophisticated understanding of how occupants interact with their surrounding environments if they are to successfully decrease inadvertent occupant related energy consumption. It is clear that if governments around the world are to meet their ambitious emissions targets then designers cannot continue to marginalise the building occupants during the design process and a more user-centered approach must be adopted.

\section{Acknowledgements}

The corresponding author would like to thank the EPSRC and AECOM for funding this research.

\section{References}

[1] Climate Change Act, 2008. Carbon Targeting and Budgeting, Chapter 27, Part 1 - The Target for 2050. UK: Her Majesty's Stationery Office Limited.

[2] BIS (Department for Business, Innovation and Skills) 2010. Low Carbon Construction, Innovation and growth team: Emerging Findings. BIS, 2010.

[3] CarbonBuzz, 2013. Website: http://www.carbonbuzz.org

[4] Bordass, B., Cohen, R. and Field, J., 2004. Energy Performance of Non-Domestic Buildings - Closing the Credibility Gap, International Conference on Improving Energy Efficiency in Commercial Buildings. Frankfurt, Germany.

[5] Mulville, M., Jones, K., \& Huebner, G. 2014. The potential for energy reduction in UK commercial offices through effective management and behaviour change. Architectural Engineering and Design Management 10, 79-90.

[6] Combe, N., Harrison, D., Dong, H., Craig, S. \& Gill, Z. 2010. Assessing the number of users who are excluded by domestic heating controls. International Journal of Sustainable Engineering 4, 84-92.

[7] Fabi, V., Andersen, R.V., Corgnati, S., \& Olesen, B.W. 2012. Occupants' window opening behaviour: A literature review of factors influencing occupant behaviour and models. Building and Environment 58, 188-198.

[8] Masoso, O.T., \& Grobler, L.J. 2010. The dark side of occupants' behaviour on building energy use. Energy and Buildings 42, 173-177. 
[9] BRECSU, 2000. Energy Consumption Guide 19: Energy use in offices. Watford: Building Research Energy Conservation Support Unit.

[10] CIBSE, 2009. CIBSE Guide H: Building Control Systems. Second edition. London: Chartered Institution of Building Service Engineers.

[11] Slater, A.I., Bordass, W.T., \& Heasman, T.A. 1996. People and lighting controls. BRE IP6/96. Garston: Building Research Establishment.

[12] Becker, G. S. 1976. The economic approach to human behaviour. Chicago: University of Chicago Press.

[13] Sheeran, P. 2002. Intention-behavior relations: A conceptual and empirical review. European Review of Social Psychology 12, 1-36 DOI:10.1080/14792772143000003

[14] Kollmuss, A. \& Agyeman, J. 2002. Mind the Gap: Why do people act environmentally and what are the barriers to pro-environmental behaviour? Environmental Education Research 8(3), 239-260.

[15] Owens, S. \& Driffill, L. 2008. How to change attitudes and behaviours in the context of energy. Energy Policy 36, 4412-4418.

[16] Byrne, M.D., \& Bovair, S. 1997. A working memory model of a common procedural error. Cognitive Science 21(1), 31-61.

[17] Everett, S.P., Greene, K.K., Byrne, M.D., Wallach, D.S., Derr, K., Sandler, D., \& Torous, T. 2008. Electronic voting machines versus traditional methods: Improved preference, similar performance. In Human Factors in Computing Systems: Proceedings of CHI 2008, 883-892. New York: ACM

[18] Ament, M.G.A., Lai, A.Y.T., \& Cox, A.L. 2011. The effect of repeated cue exposure on post-completion errors. In: Carlson, L., Hoelscher, C., \& Shipley, T. F. (Ed.s).

Proceedings of the 33rd Annual Conference of the Cognitive Science Society. Austin, TX: Cognitive Science Society, 850-855.

[19] Hunt, D.R.G., 1979. The use of artificial lighting in relation to daylight levels and occupancy. Building and Environment 14, 21-33.

[20] Love, J.A. 1998. Manual switching patterns observed in private offices. Lighting Research \& Technology 30(1), 45-50.

[21] Pigg, S., Eilers, M. \& Reed, J. 1996. Behavioural aspects of lighting and occupancy sensors in private offices: A case study of a university office building. Proceedings of the 1996 ACEEE summer study on energy efficiency in buildings, 161-168.

[22] Reinhardt, C.F. 2004. Lightswitch 2002: A Model for Manual Control of Electric Lighting and Blinds. Solar Energy 77, 15-28.

[23] Reinhardt, C.F., Voss, K. 2003. Monitoring manual control of electric lighting and blinds. Lighting Research \& Technology 35(3), 243-260.

[24] Fabi, V., Camisassi, V., Causone, F., Corgnati, S.P., \& Anderson, R. 2014. Light switch behaviour: occupant beahviour stochastic models in office buildings. Proceedings of $8^{\text {th }}$ Windsor Conference 2014, London: Network for Comfort and Energy Use in Buildings.

[25] Chung, P. H., \& Byrne, M. D. 2004. Visual cues to reduce errors in a routine procedural task. In Forbus, K., Gentner, D., \& Regier, T. (Ed.s).Proceedings of the 26th Annual Conference of the Cognitive Science Society. Austin, TX: Cognitive Science Society.

[26] Chung, P. H., \& Byrne, M. D. 2008. Cue effectiveness in mitigating post-completion errors in a routine procedural task. International Journal of Human-Computer Studies 66, 217-232.

[27] Byrne, M. D. 2008. Preventing post-completion errors: How much cue is enough? In: Proceedings of the 30th Annual Conference of the Cognitive Science Society, 351-356.

[28] Nolan, J., Schultz, P., Cialdini, R., Goldstein, N., \& Griskevicius, V. 2008. Normative Social Influence is Underdetected. Personality and Social Psychology Bulletin 34(7), 913923.

[29] Schultz, P. W., Nolan, J., Cialdini, R., Goldstein, N., \& Griskevicius, V. 2007. The constructive, destructive, and reconstructive power of social norms. Psychological Science $18,429-434$. 
[30] Goldstein, N., Cialdini, R., \& Griskevicius, V. 2008. A Room with a Viewpoint: Using Social Norms to Motivate Environmental Conservation in Hotels. Journal of Consumer Research, 35(3), 472-482.

[31] Howell, D.C. 2012. Statistical methods for psychology. Eight Edition. Sussex: Wadsworth.

[32] Cohen, J. 1992. A power primer. Psychological Bulletin, 112(10), 155-159.

[33] Menezes, A.C., Tetlow, R., Beaman, C.P., Cripps, A., Bouchlaghem, D., \& Buswell, R. 2012. Assessing the Impact of Occupant Behaviour on Electricity Consumption for Lighting and Small Power in Office Buildings. AEC 2012, Sao Paulo. 
Highlights:

- Post-completion errors can occur when occupants use building controls leading to wasted energy

- It is likely that PIR sensors in meeting rooms do not achieve their intended energy savings

- Normative prompts are more effective at encouraging users to turn off light than standard prompts 